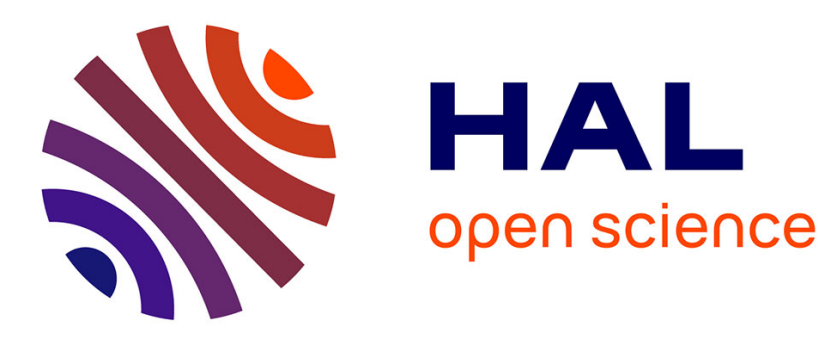

\title{
Double diffusive instability in an inclined cavity
}

Alain Bergeon, Kassem Ghorayeb, Abdelkader Mojtabi

\section{To cite this version:}

Alain Bergeon, Kassem Ghorayeb, Abdelkader Mojtabi. Double diffusive instability in an inclined cavity. Physics of Fluids, 1999, 11 (3), pp.549-559. 10.1063/1.869929 . hal-01881211

\section{HAL Id: hal-01881211 \\ https://hal.science/hal-01881211}

Submitted on 25 Sep 2018

HAL is a multi-disciplinary open access archive for the deposit and dissemination of scientific research documents, whether they are published or not. The documents may come from teaching and research institutions in France or abroad, or from public or private research centers.
L'archive ouverte pluridisciplinaire HAL, est destinée au dépôt et à la diffusion de documents scientifiques de niveau recherche, publiés ou non, émanant des établissements d'enseignement et de recherche français ou étrangers, des laboratoires publics ou privés. 


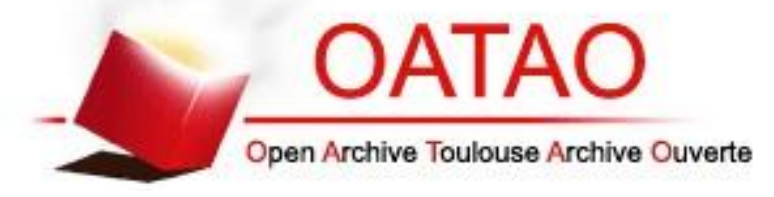

\section{Open Archive Toulouse Archive Ouverte}

OATAO is an open access repository that collects the work of Toulouse researchers and makes it freely available over the web where possible

This is an author's version published in: http://oatao.univ-toulouse.fr/20640

\section{Official URL:}

http://doi.org/10.1063/1.869929

\section{To cite this version:}

Bergeon, Alain and Ghorayeb, Kassem and Mojtabi,

Abdelkader Double diffusive instability in an inclined cavity.

(1999) Physics of Fluids, 11 (3). 549-559. ISSN 1070-6631

Any correspondence concerning this service should be sent to the repository administrator: tech-oatao@listes-diff.inp-toulouse.fr 


\title{
Double diffusive instability in an inclined cavity
}

\author{
A. Bergeon, K. Ghorayeb, and A. Mojtabi \\ U.M.R. 5502 IMFT-CNRS-UPS, U.F.R. M.I.G., 118, route de Narbonne, 31062 Toulouse Cedex, France
}

(Received 17 June 1997; accepted 30 November 1998)

\begin{abstract}
Double diffusive convection in a rectangular two-dimensional cavity with imposed temperatures and concentrations along two opposite sidewalls is considered. The study is performed for two-dimensional cavities in which the thermal and solutal buoyancy forces have the same magnitude, but are of opposite sign. The influence on the convective instability of the aspect ratio $A$ (height/length) of the cavity and the cavity inclination $\alpha$ with respect to gravity is discussed. The onset of convection is computed for an infinite layer and compared to that for bounded boxes. The study is completed by the continuation of bifurcating solutions. It is found that, due to centrosymmetry, steady bifurcations are either pitchfork or transcritical depending on $A$ and $\alpha$. However, a primary pitchfork bifurcation is found to create unstable steady solutions, even if it is the first bifurcation. For the aspect ratios we studied, and close to the onset of convection, the stable solutions are mainly one-roll structures that can be destabilized by further interactions with asymmetric solutions created at primary pitchfork bifurcations. For large aspect ratios, additional asymmetric one-roll solutions are created via more complex branch interactions. (C) 1999 American Institute of Physics. [S1070-6631(99)01603-7]
\end{abstract}

\section{INTRODUCTION}

Double diffusive convection is the name given to convective motions where two factors affect the fluid density and that typically have different rates of diffusion. The archetypical example is heat and salt in water. This field originated in oceanography $\left(\mathrm{Schmidt}^{1}\right)$, but its applications are wide, including problems related to geology, metallurgy, and crystal growth (Turner, ${ }^{2-4}$ Huppert and Turner, ${ }^{5}$ Gebhart et al., ${ }^{6}$ and $\left.\mathrm{Wilcox}^{7}\right)$.

In a typical configuration, temperature and concentration gradients are imposed across a box filled with a nonreactive binary fluid mixture. Any difference between the thermal and solutal diffusivities $\chi$ and $D$ may cause convective instabilities, even if the system is initially gravitationally stable. The resulting convective motions are varied and mostly depend on how the initial temperature and concentration gradients are oriented relative to one another. The present paper is a study of convective instabilities arising in boxes, where initial heat and mass stratifications are induced by specified temperatures and concentrations along two opposite sidewalls.

Two situations are generally considered depending on the sign of the buoyancy ratio $N=\mathrm{Gr}_{S} / \mathrm{Gr}_{T}$. Here, $\mathrm{Gr}_{S}$ $=g \beta_{C}(\Delta C) L^{3} / \nu^{2}$ and $\mathrm{Gr}_{T}=g \beta_{T}(\Delta T) L^{3} / \nu^{2}$ are the solutal and thermal Grashof numbers measuring the magnitude of the solutal and thermal buoyancy contributions, respectively. $L, \Delta C, \Delta T, g, \beta_{C}, \beta_{T}$, and $\nu$ are the width of the cavity, the concentration and temperature differences, the gravitational constant, the solutal and thermal expansion coefficients, and the kinematic viscosity, respectively. The two situations are referred to as the "cooperating case" if $N>0$, and as the " 'opposing case", if $N<0$.

The existence of multicellular flows has been widely discussed in the literature. Wang et al., ${ }^{8}$ Kamotani et al., ${ }^{9}$ and
Jiang et al. ${ }^{10,11}$ experimentally investigated low aspect ratio rectangular enclosures. Kamotani et al. ${ }^{9}$ used an electrochemical method based on a diffusion-controlled electrode reaction. For $\mathrm{Le}=300$ ( $\mathrm{Le}$ is the Lewis number defined by $\mathrm{Le}=\mathrm{Sc} / \mathrm{Pr}=\chi / D$, where $\operatorname{Pr}$ and $\mathrm{Sc}$ are the Prandtl and the Schmidt numbers, respectively), they observed unicellular flows for $-10<N<6$ and three-layer structures outside this range. Lee et al. ${ }^{12}$ studied the same situation using a membrane with supply reservoirs designed to maintain an imposed concentration and temperature along the sidewalls. For larger thermal Rayleigh numbers $\left(\mathrm{Ra}=\operatorname{Pr} \mathrm{Gr}_{T}\right)$ and Lewis numbers of order 100, they reported results that contradicted Kamotani et al. ${ }^{9}$ concerning the flow structure in various ranges of buoyancy ratio. In the same range of parameters, Lee and Hyun ${ }^{13}$ and Hyun and Lee ${ }^{14}$ conducted numerical simulations and obtained flow characteristics in good agreement with Lee et al. ${ }^{12}$ In the opposing case $(N<0)$, Jiang et al. ${ }^{11}$ also observed unsteady mass transfer processes and a very thin solutal boundary layer for large Lewis numbers. Very recently, Bennacer and Gobin ${ }^{15}$ and Gobin and Bennacer ${ }^{16}$ numerically focused on heat and mass transfer in the cooperating case. Their results have shown that, depending on the range of parameters, unicellular and multicellular steady-state flows appear.

The above studies used large Lewis number binary fluids. A large amount of work on double diffusive instabilities with moderate Lewis numbers (gas media) has also appeared in the literature. Weaver and Viskanta ${ }^{17}$ experimentally and numerically investigated this situation in a square cavity for $0.59<\mathrm{Le}<2$ and $-9.42<N<0.55$. They observed good agreement with the experimental results in the cooperating case. But due to the unsteadiness of the flow, they did not obtain agreement in the opposing case. Béghein et al. ${ }^{18} \mathrm{nu}-$ merically obtained some correlations concerning mass and 
heat transfers in a square cavity filled with air mixed with different kinds of pollutants. The comparison between the numerical results obtained by Mahajan and Angirasa ${ }^{19}$ in binary gases $(\mathrm{Le} \approx 7)$ and the theoretical results predicted by Gebhart and Pera ${ }^{20}$ by means of the thermosolutal boundary layer theory indicates that boundary layer analysis is suitable only when the two buoyant forces cooperate. ${ }^{19}$

In the present work we study steady solutions arising with exactly opposing buoyancy forces, that is, $N=-1$. It is well established that when the coefficients $D$ and $\chi$ are equal, the trivial no-flow solution, remains stable, whatever the imposed temperature difference. But when they are different, there exists a critical temperature (or concentration) difference above which the trivial solution is unstable, and some perturbations may grow. This threshold is estimated in terms of the (thermal) Rayleigh number.

Linear analyses have been performed in order to evaluate this critical Rayleigh number $\mathrm{Ra}_{c}$. Gobin and Bennacer ${ }^{21}$ established that in an infinite vertical layer, the stability of the motionless solution only depends on $\mathrm{Ra}(\mathrm{Le}-1)$. They showed, following the analysis carried out by Thorpe et al., ${ }^{22}$ that $\mathrm{Ra}_{c}=6122 /(\mathrm{Le}-1)$. The analytical solution they obtained only satisfies the impermeability condition at the sidewalls. Ghorayeb and Mojtabi ${ }^{23}$ and Xin et al. ${ }^{24}$ refined this analysis and established that $\mathrm{Ra}_{c}=6509 /(\mathrm{Le}-1)$, corresponding to the critical dimensionless wave number $k_{c}$ $=2.53$. More precisely, Ghorayeb and Mojtabi ${ }^{23}$ considered vertical two-dimensional bounded boxes with various finite extensions. Their results report the evolution of the critical Rayleigh number with the aspect ratio. Using direct numerical simulations of convective solutions, they observed the existence of a wide variety of steady one-cell and multicell solutions for a fixed aspect ratio and Grashof number.

Our purpose here is to investigate the onset of convection and the stability of the steady flow structures. This expands on the previous work of Ghorayeb and Mojtabi, ${ }^{23}$ in which the mechanisms by which stable solutions lose stability or by which bifurcating unstable solutions regain stability were not ascertained. We thus reconsider, in detail, the linear stability analysis of vertical bounded boxes and carry out the continuation of bifurcating stable or unstable steady solutions. We next turn to the influence of the cavity inclination on the stability.

The vast literature we referred to contrasts with the number of publications on the influence of cavity inclination on the flow structures. Paliwal and Chen ${ }^{26,27}$ experimentally ${ }^{26}$ and theoretically ${ }^{27}$ studied the influence of cavity inclination in a stably stratified fluid layer subjected to a lateral thermal gradient. Their results show that the agreement between the theoretical predictions (based on linear stability analysis) and the experimental measures of the critical parameters is good. A nonlinear analysis has been carried out by Thangam et $a l^{28}$ to study the initial evolution of the double-diffusive instability in an inclined cavity. In this study we indicate that the pairs of counter-rotating cells predicted by the linear theory merge into single cells, with the sense of rotation experimentally observed. In the present paper, we also study

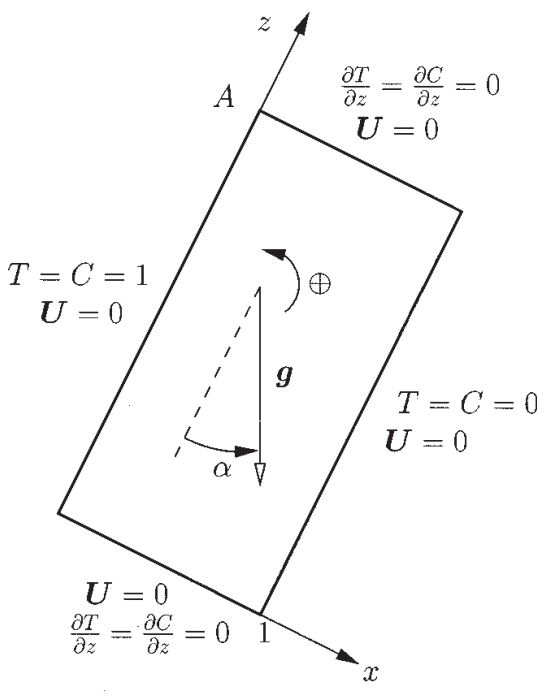

FIG. 1. Cavity configuration.

nonlinear interactions between bifurcating branches of solutions for different angles of inclination.

The paper is organized as follows. In the next section, the governing equations, their scaling, and the numerical methods are outlined. The results are divided into two parts. The first is devoted to linear analysis, where the influence of the aspect ratio and of the inclination of the box with respect to gravity are studied. The second part is the study of the bifurcating solutions. A continuation method is used that allows us to follow the path of stable or unstable steady solutions.

\section{MATHEMATICAL PROBLEM AND NUMERICAL METHODS}

\section{A. Mathematical formulation}

We consider a two-dimensional cavity of length $L$ and height $H$ and aspect ratio $A=H / L$ (Fig. 1). The box is filled with a binary, incompressible fluid mixture. The rigid left sidewall is maintained at a constant temperature $T_{0}+\Delta T$ and concentration $C_{0}+\Delta C$ and the right sidewall at $T_{0}$ and $C_{0}$, respectively $(\Delta T>0, \Delta C>0)$. We assume that the Boussinesq approximation is valid in the range of temperatures and concentrations expected, so that the density $\rho$ is constant except in the buoyancy term, where it varies linearly with the temperature and concentration:

$$
\rho(T, C)=\rho_{0}\left[1-\beta_{T}\left(T-T_{0}\right)-\beta_{C}\left(C-C_{0}\right)\right],
$$

where $\beta_{T}$ and $\beta_{C}$ are the thermal and solutal expansion coefficients respectively. In the following, we assume that $\beta_{T}$ $>0$ and $\beta_{C}<0$ ( $\rho$ increases with $C$ and decreases with $T$ ). The other physical properties, the mass diffusion coefficient $D$, the thermal diffusivity $\chi$, and the kinematic viscosity $\nu$ are constant. The time, distance, velocity $\mathbf{U}(u, w)$, temperature $T$, and concentration $C$ are scaled with $L^{2} / \nu, L, \nu / L$, $\Delta T$, and $\Delta C$, respectively. The dimensionless equations are 


$$
\begin{aligned}
\frac{\partial \mathbf{U}}{\partial t}= & -(\mathbf{U} \cdot \boldsymbol{\nabla}) \mathbf{U}-\boldsymbol{\nabla} p+\nabla^{2} \mathbf{U} \\
& +\mathrm{Gr}_{T}(T+N C)(\cos \alpha \mathbf{k}-\sin \alpha \mathbf{i}), \\
\frac{\partial T}{\partial t}= & -(\mathbf{U} \cdot \boldsymbol{\nabla}) T+\frac{1}{\operatorname{Pr}} \nabla^{2} T, \\
\frac{\partial C}{\partial t}= & -(\mathbf{U} \cdot \boldsymbol{\nabla}) C+\frac{1}{\mathrm{Sc}} \nabla^{2} C, \\
\boldsymbol{\nabla} \cdot \mathbf{U}= & 0,
\end{aligned}
$$

where $\alpha$ is the angle $(-\mathbf{k}, \mathbf{g}),(\mathbf{i}, \mathbf{k})$ are the $x$ and $z$ unit vectors, and $\operatorname{Pr}=\nu / \chi$ and $\mathrm{Sc}=\nu / D$ are the usual Prandtl and Schmidt numbers. $\mathrm{Gr}_{T}, \mathrm{Gr}_{S}$, and $N$ are the thermal and solutal Grashof numbers and the buoyancy ratio, defined as

$$
\mathrm{Gr}_{T}=\frac{g \beta_{T}(\Delta T) L^{3}}{\nu^{2}}, \quad \mathrm{Gr}_{S}=\frac{g \beta_{C}(\Delta C) L^{3}}{\nu^{2}}, \quad N=\frac{\mathrm{Gr}_{S}}{\mathrm{Gr}_{T}},
$$

where $g$ is the gravitational constant. No-slip conditions are imposed along the rigid wall. Across the lower and upper boundaries, the normal heat and mass fluxes are zero. Accordingly, the set of boundary conditions is

$$
\begin{aligned}
& \text { at } z=0 \quad \text { and } z=A, \quad u=w=\frac{\partial C}{\partial z}=\frac{\partial T}{\partial z}=0 ; \\
& \text { at } x=0, \quad u=w=T-1=C-1=0 ; \\
& \text { at } x=1, \quad u=w=T=C=0 .
\end{aligned}
$$

In the following, we restrict our study to the case $N=$ -1 and we denote $\mathrm{Gr}=\mathrm{Gr}_{T}=-\mathrm{Gr}_{S}$. The problem has the following purely diffusive solution:

$$
\mathbf{U}_{d}=0, \quad T_{d}(x)=C_{d}(x)=1-x .
$$

\section{B. Numerical methods}

We used two numerical methods to investigate the problem: time integration and a continuation method. Time integration was carried out using a pseudospectral method. The fields are discretized using the spectral element method $\left(\right.$ Patera $\left.^{29}\right)$ with Chebyshev polynomials along the LobattoChebyshev points. The linear terms are integrated implicitly and the nonlinear terms explicitly using the pressure boundary conditions given by Karniadakis. ${ }^{30}$ The resulting Poisson problem on the pressure and the Helmoltz problems arising from the diffusion terms are solved using a variational formulation.

The continuation method uses the time integration scheme that is adapted to compute steady states with a Newton method. ${ }^{31-34}$ The linear Newton system is solved using an iterative conjugate gradient method. Once a solution has been computed, classical continuation is performed, enabling us to follow any branch of steady solutions. Finally, we occasionally compute the leading eigenvalues and their corresponding eigenvectors. ${ }^{32}$ The Newton solver has been adapted to compute primary bifurcation points and to follow their evolution with the aspect ratio and the inclination angle. $^{33,34}$

\section{RESULTS}

The linear analysis of the diffusive solution shows that the threshold of instability depends on the product $\mathrm{Ra}(\mathrm{Le}$ -1 ), where $\mathrm{Ra}$ is the usual (thermal) Rayleigh number. Note that with the choice of the Rayleigh number Ra as a dimensionless parameter, the threshold does not depend on the Prandtl number. All the linear results will be thus presented in terms of the thermal Rayleigh number instead of the thermal Grashof number.

For the nonlinear behavior, we keep the Grashof number for the bifurcation diagrams. The values of the Grashof number at secondary bifurcations or saddle-node points will depend on the Prandtl and Schmidt numbers. However, we believe that small variations in Pr or Sc do not produce important qualitative changes in the bifurcation diagrams we present. The next results are obtained for $\operatorname{Pr}=1$ and $\mathrm{Sc}=11$. This choice is motivated by earlier results (Ghorayeb and Mojtabi ${ }^{23}$ ). A Prandtl number of the order of 1 and a Schmidt number of the order of 10 to 100 correspond to molten salts (Henry and Roux ${ }^{35}$ ).

\section{A. Linear analysis}

In this section, we present the results of the linear stability of the motionless solution. The vertical infinite box is the next subsection and it is followed by the study of the bounded box. The equations we consider are obtained by linearizing Eqs. (2) and (4) about the purely diffusive solution (5). The resulting eigenvalue problem is

$$
\begin{aligned}
& \lambda \mathbf{U}^{\prime}=-\nabla p^{\prime}+\nabla^{2} \mathbf{U}^{\prime}+\operatorname{Gr}\left(T^{\prime}-C^{\prime}\right)(\cos \alpha \mathbf{k}-\sin \alpha \mathbf{i}), \\
& \lambda T^{\prime}=\mathbf{U}^{\prime}+\frac{1}{\operatorname{Pr}} \nabla^{2} T^{\prime}, \\
& \lambda C^{\prime}=\mathbf{U}^{\prime}+\frac{1}{\mathrm{Sc}} \nabla^{2} C^{\prime}, \\
& \boldsymbol{\nabla} \cdot \mathbf{U}^{\prime}=0,
\end{aligned}
$$

with the boundary conditions

$$
\begin{aligned}
& \text { at } z=0 \quad \text { and } z=A, \quad u^{\prime}=w^{\prime}=\frac{\partial C^{\prime}}{\partial z}=\frac{\partial T^{\prime}}{\partial z}=0 \\
& \text { at } x=0 \quad \text { and } x=1, \quad u^{\prime}=w^{\prime}=T^{\prime}=C^{\prime}=0,
\end{aligned}
$$

where $\mathbf{U}^{\prime}, p^{\prime}, T^{\prime}$, and $C^{\prime}$ are the velocity, pressure, temperature, and concentration perturbations. Here $\lambda$ is the eigenvalue. At a primary steady bifurcation, $\lambda=0$. These equations indicate that $C^{\prime}=\operatorname{Le} T^{\prime}$. Eliminating $C^{\prime}$ in the equations, it is easily seen that the control parameter is $\mathrm{Ra}(\mathrm{Le}-1)$.

\section{Infinite layer}

For the infinite vertical layer, boundary conditions (7) are replaced by periodic boundary conditions of an imposed wave number $k$. Equations (6) are rewritten using a streamfunction $\left(\psi^{\prime}\right)$ formulation and the $z$ dependence of the fields $\psi^{\prime}, T^{\prime}$, and $C^{\prime}$ is $e^{i k z}$. The resulting system is a sixth-order differential equation with six boundary conditions. It is 

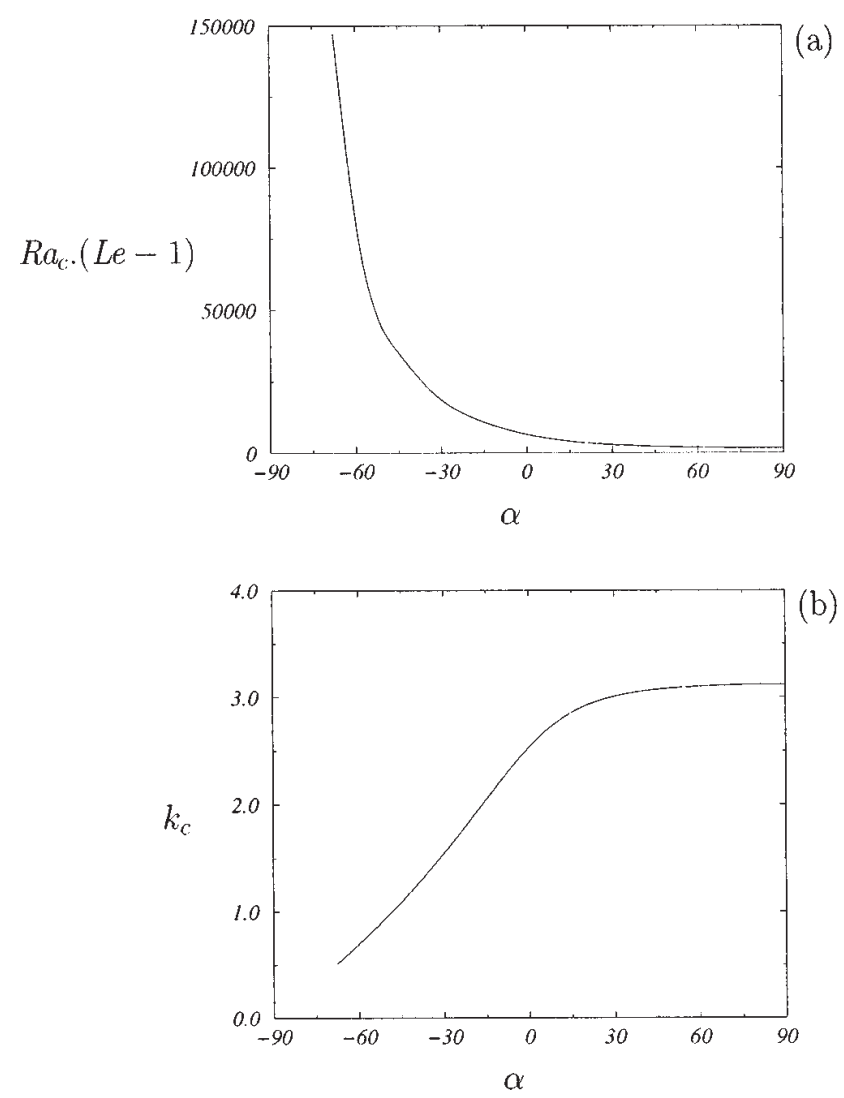

FIG. 2. Infinite layer: critical product $\mathrm{Ra}_{c}(\mathrm{Le}-1)$ (a) and critical wave number $k_{c}$ (b) versus the inclination $\alpha$.

solved by the compound matrix method already used successfully by Ghorayeb and Mojtabi. ${ }^{23}$ The results are displayed in Figs. 2(a) and 2(b), and in Table I, in which the variations of $\operatorname{Ra}_{c}(\mathrm{Le}-1)$ and the critical wave number $k_{c}$ with the inclination are presented.

At $\alpha=0$, the threshold is $\operatorname{Ra}_{c}(\mathrm{Le}-1)=6509.03$, and the critical wave number is $k_{c}=2.5321$. When $\alpha$ increases from 0 to $\pi / 2$, the critical Rayleigh number decreases monotonically to $\operatorname{Ra}_{c}(\mathrm{Le}-1)=1707.76$ with $k_{c}=3.1156$. At $\alpha=\pi / 2$, the cavity is horizontal. The fluid layer has a higher temperature and concentration at the top. In the absence of the temperature difference, the problem reduces to the RayleighBénard instability, in which the role of the temperature gradient is played by the concentration gradient. When the

TABLE I. Infinite layer: Variation of $\mathrm{Ra}_{c} \cdot(\mathrm{Le}-1)$ and $k_{c}$ with $\alpha$.

\begin{tabular}{lcccrc}
\hline \hline \multicolumn{1}{c}{$\alpha$} & $\mathrm{Ra}_{c} \cdot(\mathrm{Le}-1)$ & $k_{c}$ & \multicolumn{1}{c}{$\alpha$} & $\mathrm{Ra}_{c} \cdot(\mathrm{Le}-1)$ & $k_{c}$ \\
\hline 0 & 6509.03 & 2.5321 & & & \\
$+\pi / 16$ & 4605.99 & 2.7923 & $-\pi / 16$ & 9493.00 & 2.1761 \\
$+\pi / 12$ & 4147.39 & 2.8537 & $-\pi / 12$ & 10795.00 & 2.0490 \\
$+\pi / 8$ & 3430.82 & 2.9447 & $-\pi / 8$ & 14086.00 & 1.7916 \\
$+\pi / 6$ & 2916.11 & 3.0048 & $-\pi / 6$ & 18631.00 & 1.5439 \\
$+\pi / 4$ & 2272.73 & 3.0700 & $-\pi / 4$ & 35184.00 & 1.0912 \\
$+\pi / 3$ & 1930.10 & 3.0989 & $-\pi / 3$ & 81922.00 & 0.6943 \\
$+3 \pi / 8$ & 1827.26 & 3.1074 & $-3 \pi / 8$ & 147156.00 & 0.5121 \\
$+5 \pi / 12$ & 1759.65 & 3.1126 & & & \\
$+\pi / 2$ & 1707.76 & 3.1156 & & & \\
\hline \hline
\end{tabular}

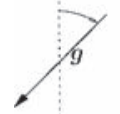

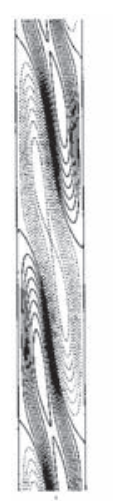
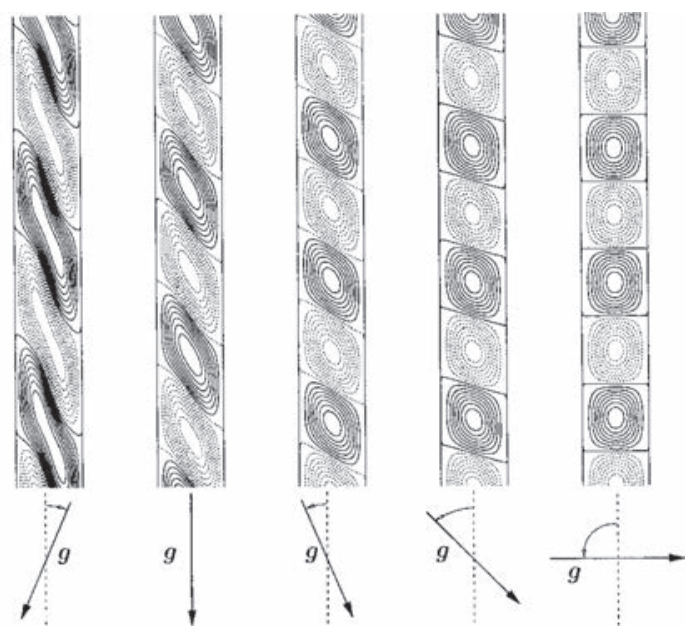

FIG. 3. Streamfunction contours of the eigenvector for the infinite layer with $\alpha=-\pi / 4$ (on the left side) $-\pi / 8,0, \pi / 8, \pi / 4$, and $\pi / 2$ (on the right). The solid and dashed lines designate clockwise and counterclockwise rotations, respectively. The cavity inclination with respect to gravity is indicated close to the insets.

temperature gradient is added, the layer becomes more stable with respect to buoyancy, and the region of stability is extended from the critical Rayleigh number $\mathrm{Ra}_{c}$ to $\mathrm{Ra}_{c}(\mathrm{Le}$ -1 ) (for $\alpha=\pi / 2$ ).

Figure 2(a) indicates that the horizontal box $(\alpha=\pi / 2)$ is far less stable than the vertical box. If the cavity is inclined in the other direction $(\alpha<0)$, the critical Rayleigh number increases to infinity and the critical wave number decreases to zero. In Fig. 3, we display the structure of the convective rolls in an arbitrary vertical extension. When $\alpha$ increases, the extension of the rolls in the direction of the gravity increases.

Without the thermal contribution, the limit $\alpha=-\pi / 2$ corresponds to a stable Rayleigh-Bénard configuration: it is stable for any finite-amplitude perturbation. When thermal effects are added, it remains linearly stable. The main reason is that the Lewis number is larger than one. When Le $<1$ and when $\alpha$ increases (decreases), the situation becomes more stable (unstable).

The simplest physical explanation of the stabilizing effect of heating from below $(\alpha<0)$ is the following. Let us consider a fluid particle $p$ lying at a distance $x_{0}$ from the warmer and more concentrated wall $(x=0)$. Suppose that the Lewis number is much larger than one and that only diffusive heat and mass transfers initially occur (purely diffusive regime). The thermal and solutal gradients thus balance each other such that the resulting density gradient vanishes. We first consider a vertical cavity.

Suppose now that the particle $p$ is moved away from the wall a distance $\delta x$ along the $x$ direction. The particle $p$ is introduced into surroundings that are cooler and at a lower concentration. The particle is thus cooled but its salinity does not change very much, owing to a smaller solutal diffusivity (Le $\gg 1)$. The density difference between the particle $p$ and its vertical surroundings is mainly due to the concentration difference and not to the temperature difference, so that the thermal contribution to the vertical density gradient can be 
negligible. This density difference drives the particle $p$ downward.

The vertical concentration gradient can be approximated by $\partial C / \partial z_{0} \approx(\Delta C / L)\left(\delta x / \delta z_{0}\right)$, where $\delta z_{0}$ is an arbitrary small distance in the vertical surroundings of $p$. If the cavity is inclined at an angle $\alpha$, the estimate of the vertical concentration gradient becomes $(\Delta C / L)$. Here $\left(\delta x+\delta_{1} x\right) / \delta z_{0}$ $\approx(\Delta C / L)\left(\delta x / \delta z_{0}+\sin \alpha\right)$, where $\delta_{1} x$ indicates that at a small vertical translation $\delta z_{0}$ of $p$ corresponds a variation $\delta_{1} x$ of its $x$ coordinate. We note that this crude estimate of the concentration gradient increases with $\alpha$. The magnitude of the solutally induced buoyancy force acting on a given perturbation becomes stronger as $\alpha$ increases. Or in other terms, for a positive inclination, a lower concentration difference $\Delta C$ is required to obtain the magnitude of the buoyancy forces in the vertical cavity. As the Rayleigh number is proportional to $\Delta C$, the critical Grashof number decreases when $\alpha$ increases from $-\pi / 2$ to $\pi / 2$. However, these physical arguments ignore the viscosity effects and consider the thermal contribution to be negligible.

The behavior of a horizontal fluid layer submitted to vertical thermal and solutal gradients has been widely discussed in the literature (Turner, ${ }^{2}$ Turner, ${ }^{4}$ and Sclunidt ${ }^{1}$ ). Linear stability analysis has been performed by Stern, ${ }^{36}$ Baines and Gill, ${ }^{37}$ Nield, ${ }^{38}$ and Veronis. ${ }^{39}$ The results they obtained are summarized in the work of Turner. ${ }^{2}$

In the situation $\alpha=\pi / 2$ and $N \neq-1$, long narrow convecting cells, called salt fingers, are predicted and have been observed over a certain range of parameters $(N, \mathrm{Le}, \mathrm{Ra})$. According to Turner (Turner, ${ }^{2}$ Fig. 2, p. 40), the situation we study $(N=-1, \alpha=\pi / 2)$ is at the limit between the salt finger regime and the steady convective regime. The convective solutions we obtained are fully within the convective regime, and there is no indication of the proximity of the finger regime. We also did not observe the oscillatory mode of instability with a stabilizing solutal gradient when the layer is heated from below. Again according to the results displayed by Turner for $N=-1$, the oscillatory mode can occur below a critical value of the thermal Rayleigh number that depends on Pr and Sc.

\section{Bounded layer}

We refer to the cavity in which the lateral walls are maintained at constant temperatures and concentrations as a vertical cavity. The horizontal extent of the box is taken to be 1 , so that its vertical extent is the aspect ratio $A$.

For a given geometry, the critical Rayleigh number depends on the Lewis number with a relation of the type: $\mathrm{Ra}_{c}(\mathrm{Le}-1)=K(A, \alpha)$. In the previous section, we obtained $K=6509.3$ for an infinite vertical layer $(\alpha=0$ and $A \rightarrow \infty)$. Figure 4 displays the evolution of $K$ with the cavity aspect ratio at the two first bifurcation points. The lowest value $\mathrm{Ra}_{c}$ is the value above which arbitrarily, infinitesimally small perturbation may grow. It does not correspond to the threshold below which convection cannot appear. As previously mentioned, the critical Rayleigh number depends on the Lewis number and the aspect ratio. Here, for instance, we find that $\mathrm{Ra}_{c}=17172 /(\mathrm{Le}-1)$ for a square cavity.

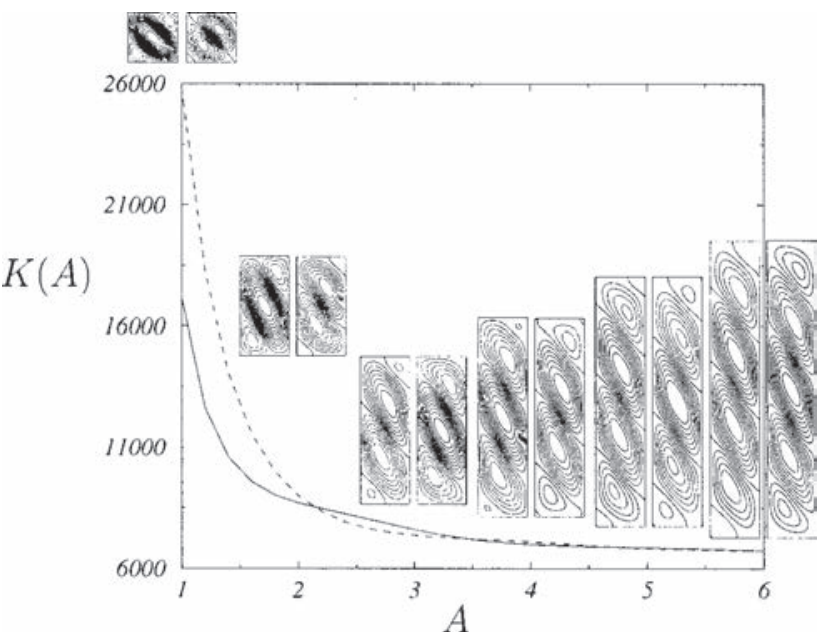

FIG. 4. The critical product $K(A, \alpha=0)=\operatorname{Ra}_{c}(\mathrm{Le}-1)$ for the two first steady bifurcations versus the aspect ratio. The box is vertical $(\alpha=0)$. The solid curve represents transcritical bifurcations and the dashed curve, pitchfork bifurcations. Insets are streamfunction contours of the null eigenvectors for integer aspect ratios. The null eigenvector of the first bifurcation is drawn on the left side.

The geometry and the set of equations linearized about the purely diffusive solution are invariant under centrosymmetry, i.e., rotation of $180^{\circ}$ about the midpoint of the cavity. Let $S_{0}$ denote this centrosymmetry and $\mathbf{X}(u, w, T, c)$ a steady solution. The operation $S_{0}: \mathbf{X} \rightarrow S_{0}(\mathbf{X})$ is $(u, v, T, C)(x, z) \rightarrow$ $-(u, v, T, C)(1-x, A-z)$. One can easily prove that all of the eigenvectors are either $S_{0}$ symmetric, that is, $\left(u^{\prime}, v^{\prime}, T^{\prime}, C^{\prime}\right)(x, z)=-\left(u^{\prime}, v^{\prime}, T^{\prime}, C^{\prime}\right)(1-x, A-z)$ or $S_{0}$ antisymmetric, that is, $\left(u^{\prime}, v^{\prime}, T^{\prime}, C^{\prime}\right)(x, z)$ $=\left(u^{\prime}, v^{\prime}, T^{\prime}, C^{\prime}\right)(1-x, A-z)$. Symmetric eigenvectors contain an odd number of cells and antisymmetric eigenvectors contain an even number of cells. We observe that the flow structure changes continuously along each bifurcation curve but that the parity remains the same (Fig. 4). The creation of an additional roll is depicted in Fig. 4 for aspect ratios varying from 1 to 6 . We observe that the new rolls are initially infinitesimally small and created in the upper right and lower left corners. All eigenvectors are either $S_{0}$ symmetric or $S_{0}$ antisymmetric. As discussed by Crawford and Knobloch, ${ }^{40}$ eigenvectors that have broken the $S_{0}$ symmetry correspond to a pitchfork bifurcation, whereas eigenvectors with $S_{0}$ symmetry correspond to transcritical bifurcations.

From Fig. 4, an estimate of the critical dimensionless wavelength of a convective roll can be obtained. The two curves of bifurcation points cross at some particular aspect ratio values. When the aspect ratio is increased above one of these values, the number of rolls of the first critical eigenvector increases by one. An estimate of the typical length of a convective roll is approximately given by the distance along the aspect ratio axis separating two successive points at which the two curves cross. Figure 4 indicates that this occurs at 2.2 and 3.5 , leading to a critical dimensionless wavelength $l_{c} \approx 2.6$.

We restricted ourselves to the two first bifurcations. To draw the curves in Fig. 4, we computed the most unstable eigenvectors at $A=1$ for various Grashof numbers and 


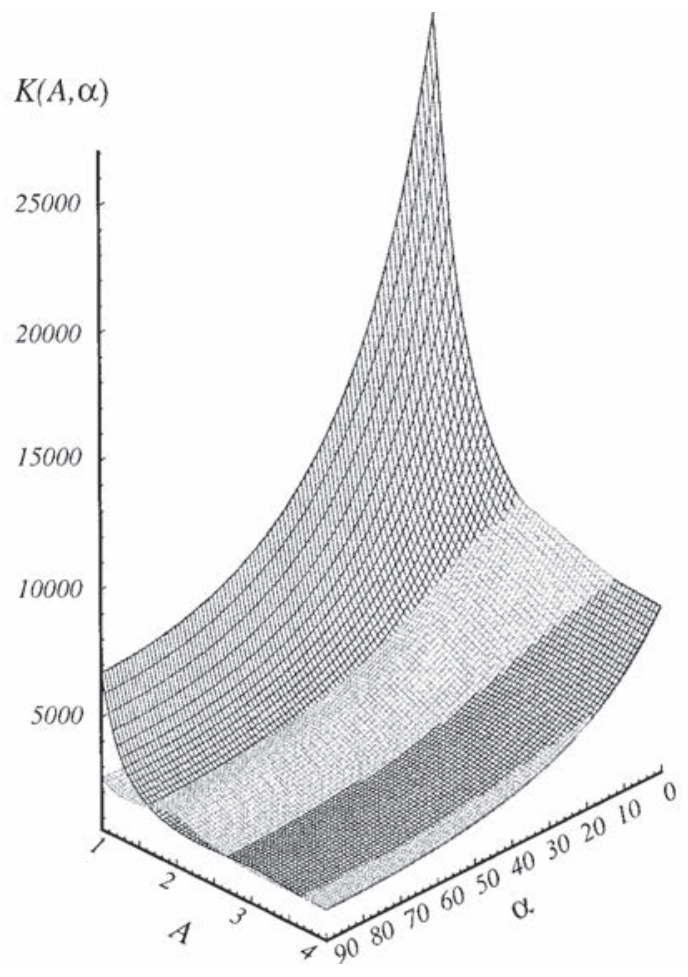

FIG. 5. The critical product $K(A, \alpha)=\mathrm{Ra}_{c}(\mathrm{Le}-1)$ for the two first steady bifurcations versus the aspect ratio $A$ and the inclination angle $\alpha$. The two surfaces intersect at codimension-two bifurcation points. One surface displays transcritical bifurcations and the other pitchfork bifurcations (see Fig. 4).

started a continuation from the two first bifurcation points. As explained by Riley and Winters, ${ }^{41}$ Winters et $a l .{ }^{42}$ and Cliffe and Winters, ${ }^{43}$ with no-slip boundary conditions, curves of eigenvectors of opposite parity cross at codimension-two primary bifurcation points, whereas sameparity curves approach one another and recede. This guarantees that no additional steady bifurcation points appear for larger aspect ratios in between the two eigenvector curves we computed.

We studied the influence of cavity inclination with respect to gravity. Figures 5 display the evolution of the two first thresholds with both the angle $\alpha$ and the aspect ratio $A$. For a fixed aspect ratio $A$, and when $\alpha$ increases, the critical Grashof number decreases. At $\alpha=\pi / 2$, the configuration is the opposite of the Rayleigh-Bénard configuration: the fluid is heated from the top, where the higher concentration is maintained. But, because the Lewis number is larger than one, this configuration is much more unstable than the vertical box configuration. For $A=1$ and when $\alpha$ decreases from 0 to $-\pi / 3$, we observe in Fig. 6 that the two bifurcation curves cross so that the first bifurcation becomes a pitchfork between $-0.11 \pi$ and $-0.24 \pi$. The critical product $\mathrm{Ra}_{c}(\mathrm{Le}$ $-1)$ also drastically increases, a result that was indicated by the linear theory in the infinite layer. On the other hand, when $\alpha$ increases from 0 to $\pi / 2$ they do not cross (see Fig. 5). For a fixed inclination $\alpha$ and when $A$ increases, the two bifurcation curves approach one another. The limit $\operatorname{Ra}_{c}(A$ $\rightarrow \infty, \alpha)$ is given in Fig. 2(a).

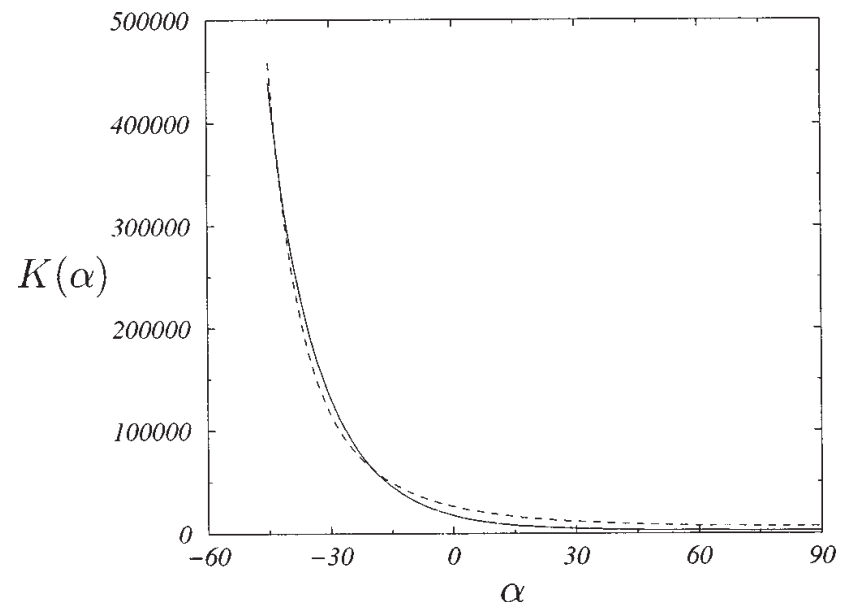

FIG. 6. The critical product $K(A=1, \alpha)=\operatorname{Ra}_{c}(\mathrm{Le}-1)$ for the two first, steady bifurcations versus the inclination angle $\alpha$ (in degrees) for a square cavity $A=1$. The solid curve represents transcritical bifurcations and the dashed curve, pitchfork bifurcations.

We mention that the results obtained by Paliwal and Chen ${ }^{26,27}$ concerning the evolution of the critical Rayleigh number qualitatively agree with our results as we show that the inclined fluid layer heated from below is much more stable than one heated from above (Le $>1$ ).

\section{B. Nonlinear study}

The results are presented with bifurcation diagrams plotting a component of the velocity at a given point of the cavity versus the Grashof number Gr. The location of this point is not important for our purposes, provided that the variations of the reported quantity with the Grashof number clearly exhibit the behavior of the whole solution. In the following, branches of stable steady solutions are continuous curves, whereas unstable solutions are dashed or dashdotted curves. Despite the fact that unstable solutions lying along the dashed curves cannot be observed physically, they indicate the way in which stable solutions are created, annihilated, or related to one another. Bifurcation diagrams aim to provide a complete map of all possible solutions over a certain range of parameter values and show the multiplicity of stable and unstable solutions. The subscripts $H, P, T, S$, and SN denote Hopf, primary pitchfork, primary transcritical, secondary pitchfork, and saddle-node bifurcation, respectively. As previously mentioned, the entire nonlinear study was conducted for $\operatorname{Pr}=1$ and $\mathrm{Sc}=11$.

\section{Vertical cavity}

For the square cavity, the purely diffusive solution is stable up to $\mathrm{Gr}_{T}=1717.2$ (Fig. 7). At $\mathrm{Gr}_{T}$, it undergoes a transcritical bifurcation. Two solution branches emerge: the stable supercritical branch for $\mathrm{Gr}>\mathrm{Gr}_{T}$ and the unstable subcritical branch for $\mathrm{Gr}<\mathrm{Gr}_{T}$. Close to $\mathrm{Gr}_{T}$, the solutions are centrosymmetric three-roll flows. At $\mathrm{Gr}_{\mathrm{SN}} \approx 676.8$, the subcritical branch undergoes a saddle-node bifurcation and becomes stable. It remains stable at least up to $\mathrm{Gr}=5000$, the end of our computational domain. On this stable part, the flow is a one-roll structure. The change from three to one roll 


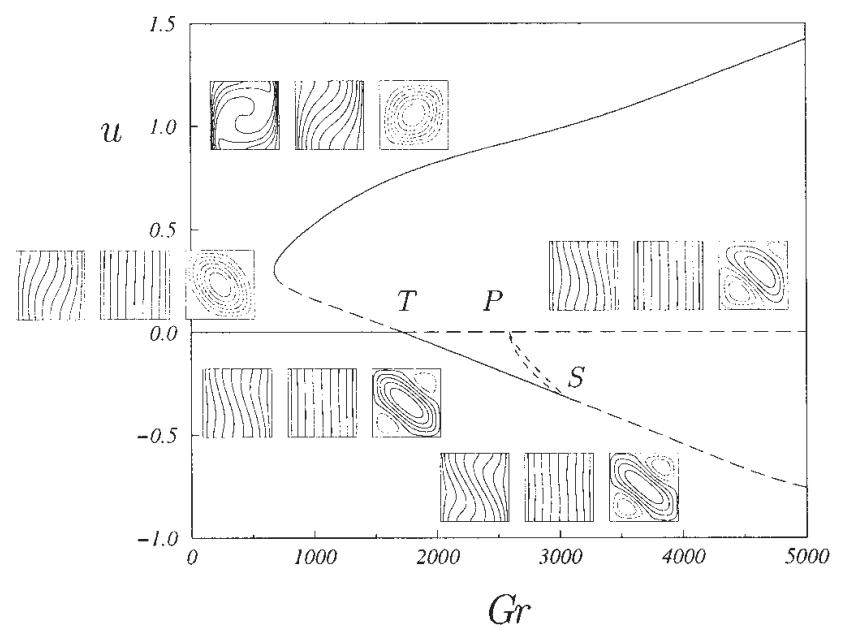

FIG. 7. The bifurcation diagram for a vertical cavity with aspect ratio $A$ $=1$. Insets are concentration, temperature, and streamfunction contours. Primary bifurcations are transcritical $(T)$ at $\mathrm{Gr}_{T}=1717$ and pitchfork $(P)$ at $\mathrm{Gr}_{T}=2589$. Secondary pitchfork bifurcation $(S)$ occurs at $\mathrm{Gr}_{S} \approx 3035$. Dashed curves denote unstable solutions and continuous lines, stable solutions. Resolution is $15 \times 15$.

occurs around the saddle-node point: the main roll grows while the two small rolls located at the upper right and lower left corners vanish. During this change, the solution remains centrosymmetric. The supercritical branch is stable up to $\mathrm{Gr}_{S}=3035$. At $\mathrm{Gr}_{S}$, it undergoes a pitchfork bifurcation. Two subcritical branches of solutions emerge for $\mathrm{Gr}<\mathrm{Gr}_{S}$. Neither solution is centrosymmetric, but for a fixed $\mathrm{Gr}$, each is transformed to the other by $S_{0}$. These two unstable branches terminate at the second primary bifurcation $\mathrm{Gr}_{P}$ $=2589.4$. When Gr increases, the inclination direction of the roll changes.

Above $A \approx 2.2$, linear stability analysis indicates that the first bifurcation is a pitchfork. Figure 8 presents the bifurcation diagram for $A=2.6$. The first bifurcation occurs for $\mathrm{Gr}_{P}=765.9$, and two subcritical unstable branches are created. With decreasing Gr, the branches approach one another and terminate at a secondary bifurcation point $\mathrm{Gr}_{S} \approx 742$. This point is located on the subcritical branch created at the second primary bifurcation for $\mathrm{Gr}_{T}=804.8$. This branch is twice unstable (i.e., it has two positive eigenvalues) down to $\mathrm{Gr}_{S}$ below which it is once unstable. For $\mathrm{Gr} \approx 503$, this branch undergoes a saddle-node bifurcation and becomes stable at least up to $\mathrm{Gr}=2000$ (the end of our computational domain). The supercritical branch emerging at $\mathrm{Gr}_{T}$ is once unstable and remains so at least up to $\mathrm{Gr}=2000$.

We observe that after the two bifurcation curves have crossed (Figs. 8 and 7), the location of the secondary bifurcation has moved from the supercritical part to the subcritical part of the transcritical bifurcation branches. This has been discussed by Riley and Winters. ${ }^{41}$

The two aspect ratios $A=1$ and $A=2.6$ show that the noncentrosymmetric solutions are unstable, even when the first bifurcation is a pitchfork. However, the influence of these branches of solution is important, as it may allow the stabilization of one of the branches emerging at the second primary bifurcation.
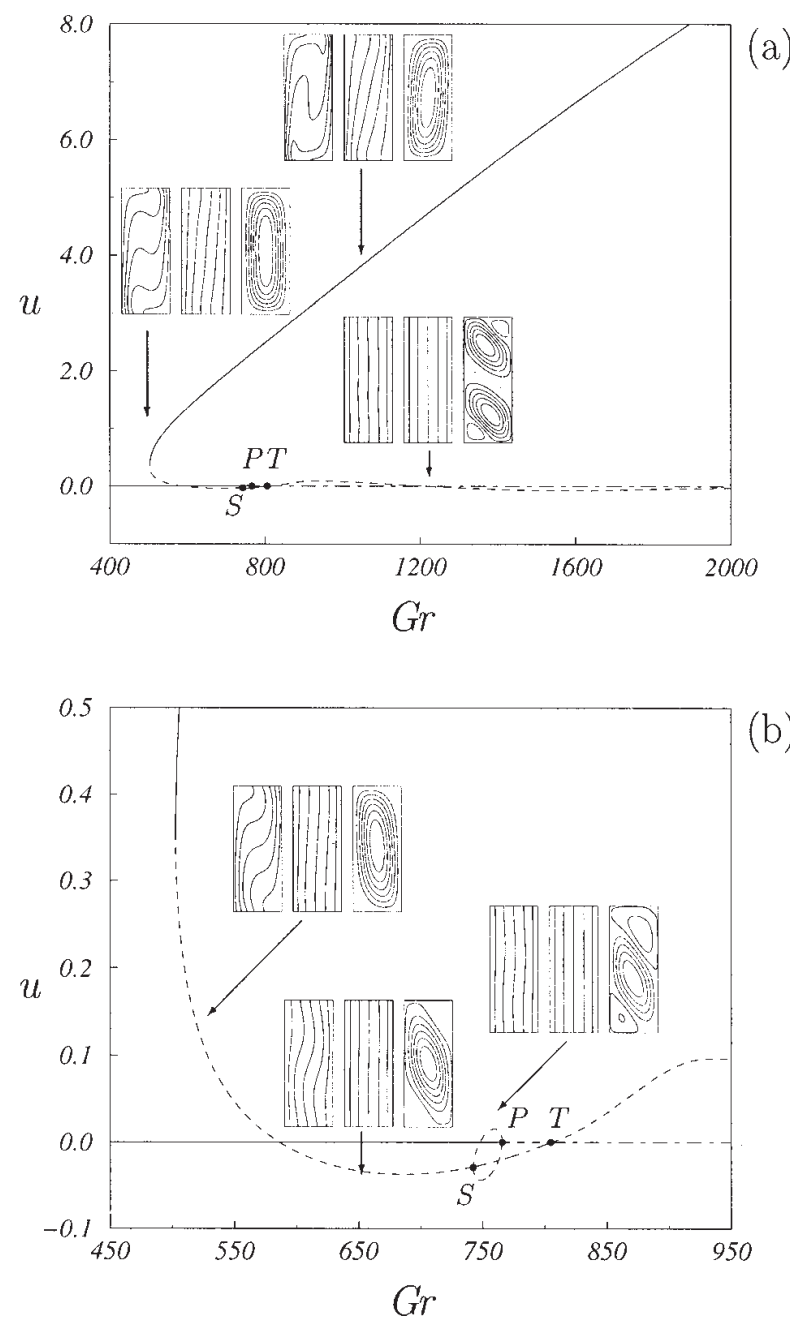

(b)

FIG. 8. (a) The bifurcation diagram for a cavity with aspect ratio $A=2.6$. Insets are concentration, temperature and streamfunction contours. Primary bifurcations are pitchfork $(P)$ to two-roll steady states at $\mathrm{Gr}_{P}=765.95$ and transcritical $(T)$ to three-roll steady states at $\mathrm{Gr}_{T}=804.76$. A secondary pitchfork bifurcation $(S)$ occurs at $\mathrm{Gr}_{S}=742.34$. Dashed curves denote onetime unstable solutions and dotted-dashed cuves two-times unstable solutions. A saddle-node occurs at $\mathrm{Gr}=502.8$. Resolution is $11 \times 21$. (b) The enlargement of (a). The bifurcating two-roll branches are destroyed at a secondary bifurcation $S$ that leaves the bifurcating three-roll branch onetime unstable for $\mathrm{Gr}<\mathrm{Gr}_{S}$.

Figure 9 displays the bifurcation diagram obtained for the aspect ratio $A=3.8$. This diagram has a complicated appearance, but the basic scenario is as before. The diffusive solution is destabilized by a transcritical bifurcation $T$ to a branch of three-roll solutions at $\mathrm{Gr}_{T}=705.5$ [Fig. 9(a)]. Along the supercritically emerging branch, a secondary pitchfork bifurcation $S_{1}$ to three-roll solutions occurs at $\mathrm{Gr}_{S 1} \approx 710.6$ and the two branches created terminate at the second primary bifurcation $P$ at $\mathrm{Gr}_{P}=715.57$. The secondary bifurcation $S_{1}$ occurs along the supercritical branch after a saddle-node bifurcation $\mathrm{SN}_{1}$. Because the saddle node destabilizes the branch for $\mathrm{Gr}<\mathrm{Gr}_{\mathrm{SN} 1} \approx 710.8$, the branch becomes twice unstable for $\mathrm{Gr}<\mathrm{Gr}_{S 1}$. The two bifurcations $\mathrm{SN}_{1}$ and $S_{1}$ are very close together so that accurate solution branches near these points cannot be computed.

Along the supercritical branch, another saddle-node bi- 


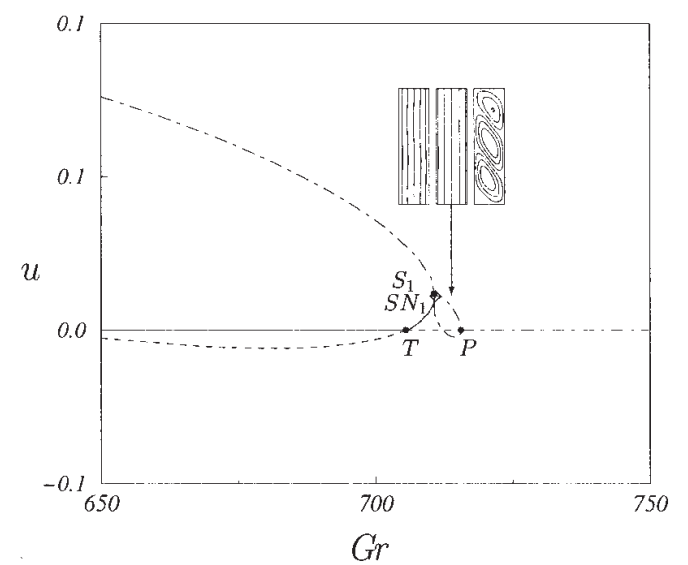

(a)

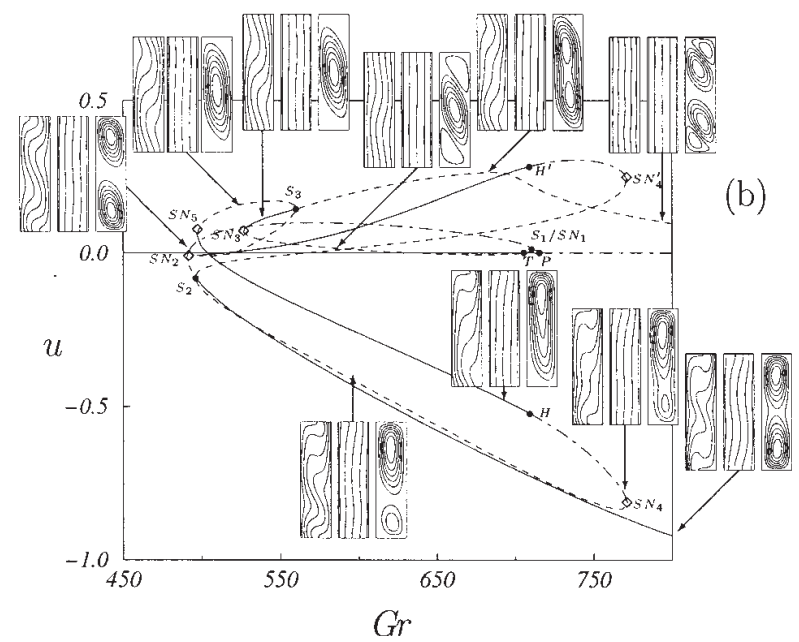

FIG. 9. (a) and (b) The bifurcation diagram for a cavity with aspect ratio $A=3$.8. Insets are concentration, temperature, and streamfunction contours. Primary bifurcations are transcritical $(T)$ to three-roll steady states at $\mathrm{Gr}_{T}$ $=705.5$ and pitchfork to four-roll structures at $\mathrm{Gr}_{P}=715.57$. Dashed curves denote one-time unstable solutions and dotted-dashed cuves two-times unstable solutions. Resolution is $11 \times 21$. (a) The enlargement of (b). The fourroll branches are destroyed at a secondary bifurcation $S_{1}$.

furcation occurs at $\mathrm{Gr}_{\mathrm{SN} 2}=490.5$ followed by a further secondary pitchfork bifurcation $S_{2}$ at $\mathrm{Gr}_{S 2}=495.8$. For $\mathrm{Gr}>\mathrm{Gr}_{S 2}$, this branch becomes stable, and the resulting flow has a two-cell structure. Each of the branches of the asymmetric two-roll states created at $S_{2}$ are once unstable. These branches undergo two successive saddle-node bifurcations at $\mathrm{Gr}_{\mathrm{SN} 4}=771.7$ and $\mathrm{Gr}_{\mathrm{SN} 5}=496.8$ and terminate at a secondary bifurcation $S_{3}$ at $\mathrm{Gr}_{S 3}=560.5$. This pitchfork bifurcation $S_{3}$ is located along the subcritical branch of one-roll solutions created at $\mathrm{Gr}_{T}$. But, surprisingly, a part of these two branches located between the two saddle nodes $\mathrm{SN}_{4}$ and $\mathrm{SN}_{5}$ is stable [see Fig. 9(b)]. Both branches undergo a Hopf bifurcation at $\mathrm{Gr}_{\mathrm{H}} \approx 710$ and become unstable. The Hopf bifurcations are supercritical and one of the two resulting stable limit cycle is shown in Fig. 10 for $\mathrm{Gr}=730$. We observe that the flow is mainly a one-roll structure whose vertical extent oscillates. The subcritical branch created at $T$ is stabilized by a saddle-node bifurcation $\mathrm{SN}_{3}$, and remains stable up to $S_{3}$.

We conclude that, compared to smaller aspect ratios, additional stable solutions have been created by secondary bi-
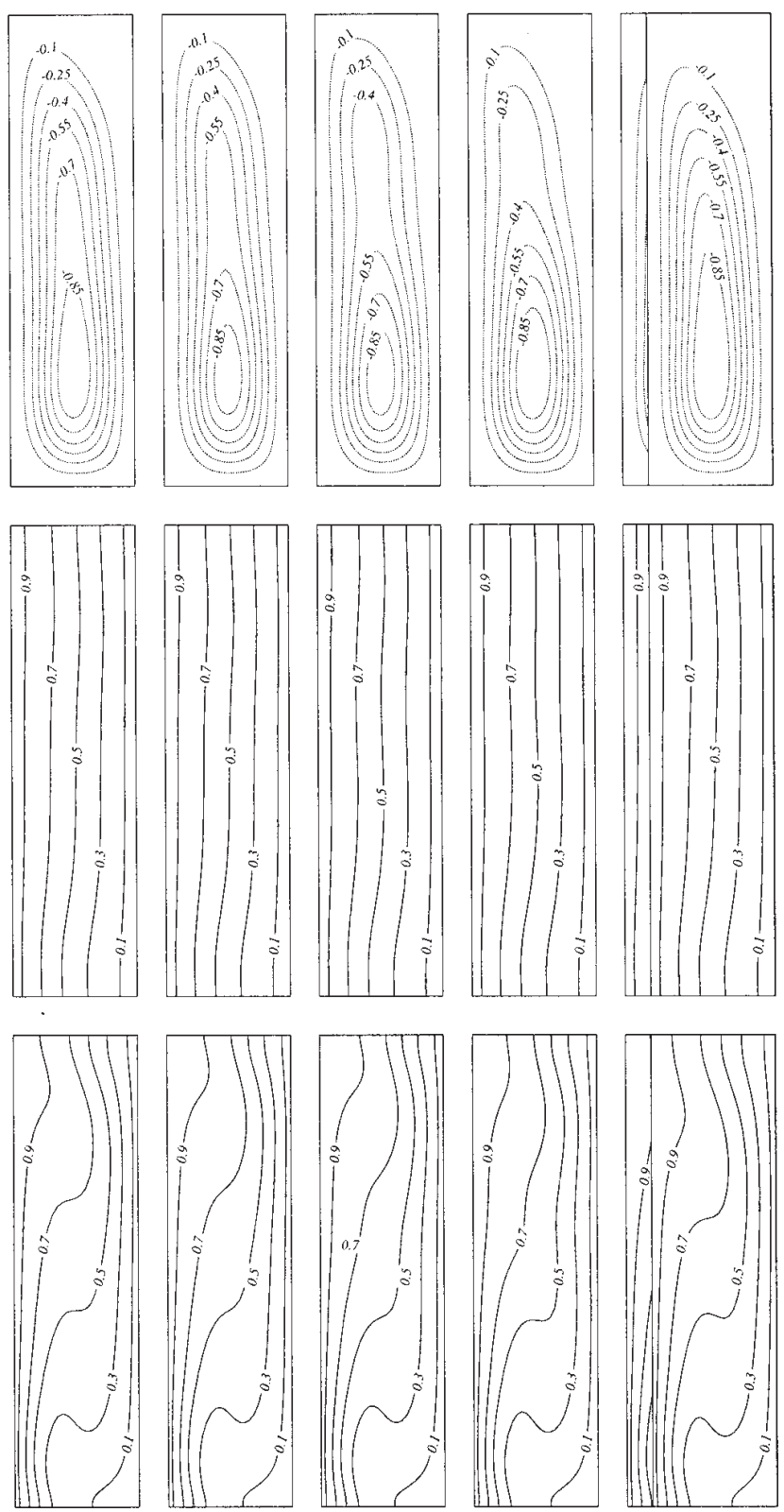

FIG. 10. The limit cycle at $\mathrm{Gr}=730, A=3.8$, and $\mathrm{Le}=11$. Contours of the streamfunction, temperature (in the middle), and concentration (bottom) fields are shown. One period is presented. Resolution is $21 \times 31$.

furcations. In particular, additional asymmetric one-roll solutions and a centrosymmetric two-roll solution have appeared. Such stable solutions have been observed by Ghorayeb and Mojtabi ${ }^{23}$ who reported similar results for $A=4$.

Similar complex sequences of bifurcations are reported in Tsitverblit's ${ }^{44}$ study of cavities with initially perpendicular gradients of temperature and concentration.

\section{Bounded inclined cavity}

We investigated the influence of the inclination on the steady solutions for the aspect ratio $A=1$. Figure 11 presents the bifurcation diagrams we obtained for $\alpha=0, \pi / 16, \pi / 8$, $3 \pi / 16$, and $\pi / 4$. For all the angles, the first bifurcation is transcritical and the second is a supercritical pitchfork bifur- 

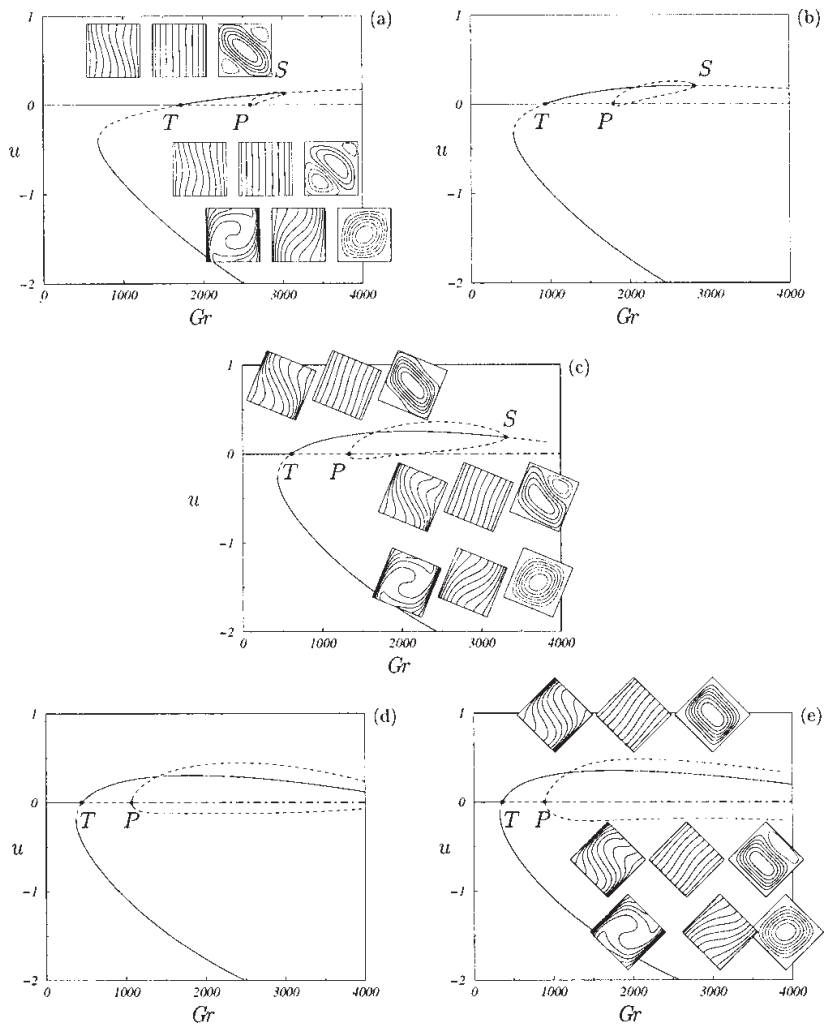

FIG. 11. Bifurcation diagrams for a cavity with $A=1$ and for $\alpha=0$ (a), $\pi / 16$ (b), $\pi / 8$ (c), $3 \pi / 16(\mathrm{~d})$, and $\pi / 4$ (e). Primary bifurcations are either pitchfork $(P)$ or transcritical $(T)$. Insets are concentration, temperature, and streamfunction contours along the supercritical branch created at $T$ (top), along one of the two branches created at $P$ (middle) and along the subcritical branch created at $T$ (bottom). As $\alpha$ is increased the hysteresis of the transcritical bifurcation $T$ decreases and the secondary bifurcation $S$ occurs at a larger Grashof number $\mathrm{Gr}_{S}$. Resolution is $15 \times 15$.

cation. When $\alpha$ increases, $\mathrm{Gr}_{T}$ approaches $\mathrm{Gr}_{\mathrm{SN}}$ and the range of existence of the subcritical branch becomes smaller. The secondary bifurcation point $S$ in Fig. 11 is the point at which the two branches of supercritical solutions emerging at $P$ are terminated. As $\alpha$ increases, $\mathrm{Gr}_{S}$ increases so that the stable part of the branch of supercritical symmetric solutions also increases. The streamfunction contours of the convective solutions are presented in Fig. 11. The limit case (Fig. 12) $\alpha=\pi / 2$ is the configuration of the Rayleigh-Bénard problem. It has the $\mathbf{Z}_{2} \times \mathbf{Z}_{2}$ symmetries that are generated by two reflections $S_{x}$ and $S_{z}$ about the $x=\frac{1}{2}$ and $z=A / 2$ axes, respectively. As discussed by Riley and Winters, ${ }^{41}$ the representation of $\mathbf{Z}_{2} \times \mathbf{Z}_{2}$ is $\Gamma=\left\{I, S_{x}, S_{z}, S_{x} S_{z}\right\}$, where $S_{x} S_{z}$ is the centrosymmetry $S_{0}$. The first bifurcation creates one-cell solutions breaking the $S_{x}$ and $S_{z}$ symmetries. The second bifurcation creates two-cell solutions breaking the $S_{x}$ and $S_{0}$ symmetries. Bifurcations $P_{1}$ and $P_{2}$ are thus pitchforks and both create two branches with $S_{0}$ and $S_{z}$ symmetric solutions, respectively. On the other hand, for a fixed Grashof, the solutions along one branch resulting from $P_{1}\left(\right.$ or $\left.P_{2}\right)$ are related by $S_{x}$ or $S_{z}$ (or by $S_{x}$ or $S_{0}$ ) to those along the other branch. Secondary bifurcations $S_{1}$ and $S_{1}^{\prime}$ occur on the twocell solution branches. Because the solutions on each branch are dynamically equivalent, this necessarily occurs at the

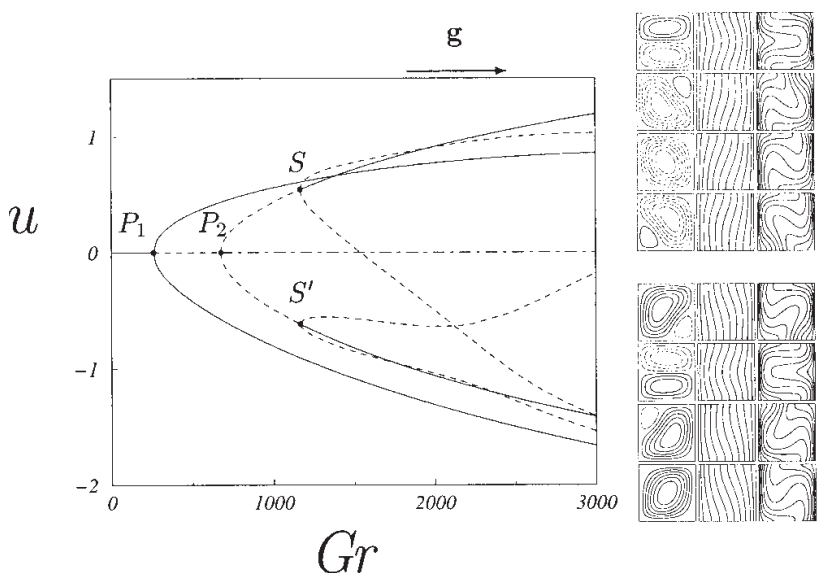

FIG. 12. The bifurcation diagram for a cavity with aspect ratio $A=1$ and an inclination $\alpha=\pi / 2$. Insets are concentration, temperature and streamfunction contours. Primary bifurcations are pitchfork $(P)$ at $\mathrm{Gr}_{P_{1}}=258.48$ and $\mathrm{Gr}_{P_{2}}$ $=674.23$. Two secondary supercritical pitchfork bifurcations $\left(S \text { and } S^{\prime}\right)^{2}$ occur at $\mathrm{Gr}_{S} \approx 1165$. Dashed curves denote one-time unstable solutions and dotted-dashed cuves two-times unstable solutions. The secondary bifurcations are pitchfork and both create two branches of asymetric solutions. The direction gravity is indicated in the figure. Resolution is $15 \times 15$.

same Grashof number. The resulting solutions break the $S_{z}$ symmetry. A similar situation is discussed by Riley and Winters ${ }^{41}$ for a porous medium. The difference is an additional translation invariance in their case resulting from the slip condition along the boundaries.

We observe that there is qualitative agreement between our results and the results of Paliwal and $\mathrm{Chen}^{26,27}$ and Thangam et al. ${ }^{28}$ concerning the sense of rotation of the convective cells. One obstacle in comparing our results to experimental works is the difficulty to attain the situation $N$ $=-1$ experimentally. However, we believe that the study of the particular situation $N=-1$ provides a stepping stone to a better understanding of much more realistic situations in which $N$ differs from -1 . The problem is qualitatively different when $N=-1+\epsilon$. The purely diffusive solution is no longer a solution and, consequently, the branch of no-flow solutions is replaced by a branch of convective solutions. A similar phenomenon is considered by Riley and Winters ${ }^{25}$ in a study of the analog of the Rayleigh-Bénard instability in a saturated porous medium. The two-dimensional horizontal configuration they consider is invariant under reflection in the vertical and horizontal middle axes, and under its product, called centrosymmetry. Thus, its symmetry group is a representation of the group $\mathbf{Z}_{2} \times \mathbf{Z}_{2}$. When the cavity is tilted, there is no longer a purely diffusive solution. The governing system of equations has lost the reflection symmetries but has kept the centrosymmetry. The authors observe that the effect of tilting the cavity is to unfold all bifurcations, except those that break the centrosymmetry.

For the vertical box that we consider here, changing $N$ does not affect the centrosymmetry and the purely diffusive state is not a solution when $N \neq-1$. Consequently, the bifurcations that do not break the centrosymmetry, i.e., the transcritical bifurcations, will be rendered imperfect by changing $N$, whereas the bifurcations that break the centrosymmetry, i.e., the pitchfork bifurcations, will be preserved. Since the 
transformation $N=-1$ to $N=-1+\epsilon$ is continuous, the flow structures of solutions for $N=-1+\epsilon$ may not strongly differ from those of the present work.

\section{CONCLUSIONS}

Numerical techniques were used to study the initiation of convection in double diffusive fluid layers. The influence of the cavity inclination with respect to gravity was studied for infinite layers and bounded cavities in the exactly opposing situation $\left(N=\mathrm{Gr}_{S} / \mathrm{Gr}_{T}=-1\right)$.

The results obtained with an infinite layer showed that when $\alpha$ decreases, the critical Grashof number and the critical wavelength increase toward infinity.

For $\alpha \in(-\pi / 2, \pi / 2)$ and a bounded cavity, the purely diffusive solution possesses $\mathbf{Z}_{2}$ centrosymmetry and the first primary bifurcation is either pitchfork or transcritical depending on the aspect ratio and the cavity inclination. We accurately computed the two first thresholds as functions of $\alpha$ and $A$.

We calculated bifurcation diagrams for vertical cavities. The results indicate that the two solution branches created at a pitchfork primary bifurcation are terminated at secondary bifurcation points that stabilize or destabilize the one-roll branches. In the cases we studied, when the first bifurcation is a pitchfork, it is always subcritical, so that it creates asymmetric unstable solutions. For larger aspect ratios of order 4, additional stable solutions appear. Those solutions are oneroll solutions but do not share the centrosymmetry. They result from secondary bifurcations along the branches of centrosymmetric solutions.

For $\alpha \in(-\pi / 2, \pi / 2)$, the only symmetry is centrosymmetry. When $\alpha$ increases from 0 to $\pi / 2$, the hysteresis induced by transcritical bifurcations decreases, and the location of the secondary bifurcation, which destabilizes the subcritical solution, approaches infinity. As a result, the region of bistability is increased. At $\alpha=\pi / 2$, the purely diffusive solution has all of the $\mathbf{Z}_{2} \times \mathbf{Z}_{2}$ symmetries, and the two first steady primary bifurcations are pitchforks. The bifurcation diagram we obtained is qualitatively the same as that obtained by Riley and Winters in the Lapwood convection problem.

\section{ACKNOWLEDGMENT}

This research was carried out within the framework of the Centre National d'Etudes Spatiales (CNES) microgravity program. The authors thank M. Karimi-Fard, H. BenHadid, D. Henry, and L.S. Tuckerman for fruitful discussions. Computations were carried out on the Cray YMP C98 and C94 of the Institut du Développement et des Ressources en Informatique Scientifique (IDRIS) sponsored by the Centre National de Recherche Scientifique (CNRS).

${ }^{1}$ R. W. Schmidt, “'Double diffusion in oceanography,'” Annu. Rev. Fluid Mech. 26, 255 (1994)

${ }^{2}$ J. S. Turner, "Double diffusive phenomena,' Annu. Rev. Fluid Mech. 6, 37 (1974).

${ }^{3}$ J. S. Turner, Buoyancy Effects in Fluids (Cambridge University Press, Cambridge, England, 1979).

${ }^{4}$ J. S. Turner, 'Multicomponent convection,'” Annu. Rev. Fluid Mech. 17, 11 (1985).
${ }^{5}$ H. E. Huppert and J. S. Turner, “Double-diffusive convection,' J. Fluid Mech. 106, 299 (1981)

${ }^{6}$ B. Gebhart, Y. Jaluria, R. L. Mahajan, and B. Sammakia, Buoyancy Induced Flows and Transport (Hemisphere, Bristol, PA, 1988).

${ }^{7}$ W. R. Wilcox, "Transport phenomena in crystal growth from solution," Prog. Cryst. Growth Charact. 26, 153 (1993).

${ }^{8}$ L. W. Wang, Y. Kamotani, and S. Ostrach, "Experimental study of natural convection in shallow horizontal cavity with different end temperatures and concentrations,' Report FATS/TR-81-164, Case Western University, 1983.

${ }^{9}$ Y. Kamotani, L. W. Wang, S. Ostrach, and H. D. Jiang, “Experimental study of natural convection in shallow enclosures with horizontal temperature and concentration gradients,' Int. J. Heat Mass Transf. 28, 165 (1985).

${ }^{10}$ H. D. Jiang, S. Ostrach, and Y. Kamotani, "Thermosolutal convection with opposed buoyancy forces in shallow enclosures," Natural Convection in Enclosures-1988, edited by R. S. Figliola and P. G. Simpkins, ASME HTD, 1988, Vol. 28, p. 165.

${ }^{11}$ H. D. Jiang, S. Ostrach, and Y. Kamotani, "'Unsteady thermosolutal transport phenomena due to opposed buoyancy forces in shallow enclosures," J. Heat Transfer 113, 135 (1991).

${ }^{12}$ J. Lee, M. T. Hyun, and K. W. Kim, "Natural convection in confined fluids with combined horizontal temperature and concentration gradients,", Int. J. Heat Mass Transf. 31, 1969 (1988).

${ }^{13}$ J. W. Lee and J. M. Hyun, "Double-diffusive convection in a rectangle with opposing horizontal temperature and concentration gradients, ' Int. J. Heat Mass Transf. 33, 1619 (1990).

${ }^{14}$ J. M. Hyun and J. W. Lee, "Double-diffusive convection in rectangle with cooperating horizontal gradients of temperature and concentration,' Int. J. Heat Mass Transf. 33, 1605 (1990).

${ }^{15} \mathrm{R}$. Bennacer and D. Gobin, "Cooperating thermosolutal convection in euclosures-I, scale analysis and mass transfer,' Int. J. Heat Mass Transf. 39, 2671 (1996).

${ }^{16} \mathrm{D}$. Gobin and R. Bennacer, "Cooperating thermosolutal convection in enclosures-II, heat transfer and flow structure,' Int. J. Heat Mass Transf. 39, 2683 (1996).

${ }^{17}$ J. A. Weaver and R. Viskanta, "Natural convection in binary gases due to horizontal thermal and solutal gradients," J. Heat Transfer 113, 141 (1991).

${ }^{18}$ C. Béghein, F. Haghighat, and F. Allard, "Numerical study of doublediffusive natural convection in a square cavity,' Int. J. Heat Mass Transf. 35, 833 (1992).

${ }^{19}$ R. L. Mahajan and D. Augirasa, "Combined heat and mass transfer by natural convection with opposing buoyancies,' J. Heat Transfer 115, 606 (1993).

${ }^{20}$ B. Gebhart and L. Pera, "The nature of vertical natural convection flows results from the combined buoyancy effects of thermal and mass diffusion,', Int. J. Heat Mass Transf. 14, 2025 (1971).

${ }^{21} \mathrm{D}$. Gobin and R. Bennacer, "Double diffusion in a vertical fluid layer; Onset of the convective regime,' Phys. Fluids 6, 59 (1994).

${ }^{22}$ S. A. Thorpe, P. P. Hutt, and R. Soulsby, "'The effect of horizontal gradients on thermohaline convection,'” J. Fluid Mech. 38, 375 (1969).

${ }^{23} \mathrm{~K}$. Ghorayeb and A. Mojtabi, "Double diffusive convection in a vertical rectangular cavity,'” Phys. Fluids 9, 2339 (1997).

${ }^{24}$ S. Xin, P. Le Quéré, and L. S. Tuckerman, "Bifurcation analysis of double-diffusive convection with opposing horizontal thermal and solutal gradients,' Phys. Fluids 10, 850 (1998).

${ }^{25}$ D. S. Riley and K. H. Winters, "A numerical bifurcation study on natural convection in a tilted two-dimensional porous cavity,' J. Fluid Mech. 215, 309 (1990).

${ }^{26}$ R. C. Paliwal and C. F. Chen, "'Double-diffusive instability in an inclined fluid layer. Part 1. Experimental investigations,'” J. Fluid Mech. 98, 755 (1980).

${ }^{27}$ R. C. Paliwal and C. F. Chen, “'Double-diffusive instability in an inclined fluid layer. Part 2. Stability analysis," J. Fluid Mech. 98, 769 (1980).

${ }^{28}$ S. Thangam, A. Zebib, and C. F. Chen, "Double diffusive convection in an inclined fluid layer,' J. Fluid Mech. 116, 363 (1982).

${ }^{29}$ A. T. Patera, "A spectral element method for fluid dynamics: Laminar flow in a channel expansion," J. Comput. Phys. 54, 468 (1984).

${ }^{30}$ G. Em. Karniadakis, M. Israeli, and S. A. Orszag, "High-order splitting method for the incompressible Navier-stokes equations,', J. Comput. Phys. 97, 414 (1991).

${ }^{31}$ L. S. Tuckerman, "Steady-state solving via Stokes preconditioning; Recursion relations for elliptic operators,' In Proceedings of the 11th Inter- 
national Conference on Numerical Methods in Fluid Dynamics, edited by D. L. Dwoyer, M. Y. Hussain, and R. G. Voigt (Springer-Verlag, Berlin, 1989).

${ }^{32}$ C. K. Mamun and L. S. Tuckerman, "Asymmetry and Hopf bifurcation in spherical Couette flow," Phys. Fluids 7, 80 (1995).

${ }^{33}$ A. Bergeon, "Instabilité de Marangoni Bénard en présence d'effet Soret,", Thesis, Ecole Centrale de Lyon, 1995.

${ }^{34}$ A. Bergeon, D. Henry, H. BenHadid, and L. S. Tuckerman, "'Marangoni convection in binary mixtures with Soret effect,' J. Fluid Mech. 375, 143 (1998).

${ }^{35}$ D. Henry and B. Roux, "Soret separation in a quasi-vertical cylinder,' J. Fluid Mech. 195, 175 (1988).

${ }^{36}$ M. E. Stern, "The salt fontain and thermohaline convection," Tellus 12, 172 (1960)

${ }^{37}$ P. G. Baines and A. E. Gill, “On thermohaline convection with linear gradients," J. Fluid Mech. 37, 289 (1969).

${ }^{38}$ D. A. Nield, "The thermohaline Rayleigh-Jeffreys problem," J. Fluid
Mech. 29, 545 (1967)

${ }^{39} \mathrm{G}$. Veronis, "Effect of a stabilizing gradient of solute on thermal convection,' J. Fluid Mech. 34, 315 (1968).

${ }^{40}$ J. D. Crawford and E. Knobloch, "Symmetry and symmetry breaking in fluid dynamics,' Annu. Rev. Fluid Mech. 23, 341 (1991).

${ }^{41}$ D. S. Riley and K. H. Winters, "Modal exchange in Lapwood convection,’ J. Fluid Mech. 204, 489 (1989).

${ }^{42}$ K. H. Winters, T. H. Plesser, and K. A. Cliffe, "The onset of convection in a finite container due to surface tension and buoyancy,', Physica D 29, 401 (1989).

${ }^{43} \mathrm{~K}$. A. Cliffe and K. H. Winters, "The use of symmetry in bifurcation calculations and its application to the Bénard problem,'” J. Comput. Phys. 67, 310 (1986).

${ }^{44} \mathrm{~N}$. Tsitverblit, "Bifurcation phenomena in confined thermosolutal convection with lateral heating: commencement of double-diffusive regime," Phys. Fluids 7, 718 (1995). 